\title{
Gestión del conocimiento y crecimiento en la Pyme manufacturera de Aguascalientes (México)
}

Knowledge management and growth in manufacturing SMEs

from Aguascalientes (Mexico)

Gonzalo Maldonado Guzmán gmaldona@correo.uaa.mx Universidad Autónoma de Aguascalientes

Doctor en Marketing, Universidad Autónoma de Aguascalientes (México), Centro de Ciencias Económicas y Administrativas, Departamento de Mercadotecnia, Coordinador del Observatorio Pyme.

María del Carmen Martínez Serna mcmartin@correo.uaa.mx Universidad Autónoma de Aguascalientes

Doctora en Administración, Universidad Autónoma de Aguascalientes (México), Centro de Ciencias Económicas y

Administrativas, Departamento de Mercadotecnia, Decana del Centro de Ciencias Económicas y Administrativas.

Ricardo García Ramírez rgarciar@correo.uaa.mx Universidad Autónoma de Aguascalientes

MBA, Universidad Autónoma de Aguascalientes (México), Centro de Ciencias Económicas y Administrativas, Departamento de Finanzas, ProfesorInvestigador.

Artículo de investigación científica y tecnológica Según Clasificación Colciencias

Fecha de recepción: $01 / 03 / 2012$

Fecha de corrección: $15 / 04 / 2012$

Fecha de aprobación: 20/05/2012
Gestion de la connaissance et croissance chez la PME manufacturière $d^{`}$ Aguascalientes (Mexique)

\section{Resumen}

En un mercado de cambios constantes y de un ambiente de los negocios incierto, el conocimiento se ha convertido en uno de los recursos intangibles más valorados y apreciados por las empresas. Por ello, cada vez son más las organizaciones que están aplicando estrategias empresariales para promover el desarrollo del conocimiento, tanto tácito como implícito, de tal manera que les permita lograr el éxito esperado. Por ello, un número importante de empresas, sobre todo pequeñas y medianas (Pymes), están incorporando la gestión del conocimiento como parte de sus actividades cotidianas, con el objetivo de mejorar su nivel de crecimiento. El valor del conocimiento está aumentando cada vez más, a tal medida que las empresas están contratando a las mentes más brillantes de cada especialidad. El objetivo de este estudio es analizar la relación existente entre la gestión del conocimiento y el crecimiento, utilizando una muestra de 125 Pymes del estado de Aguascalientes (México). Los resultados obtenidos muestran que la gestión del conocimiento tiene efectos positivos y significativos en el crecimiento de la Pyme, lo que puede constituir una ventaja competitiva.

Palabras clave: conocimiento, gestión del conocimiento, crecimiento, Pymes.

1. En el presente texto entendemos la palabra huella como los efectos no previstos de la decisión estratégica que impactan a los diferentes actores sociales de la organización según sus percepciones y experiencias. 


\section{Knowledge management and growth in manufacturing SMEs from Aguascalientes (Mexico)}

\section{Gestión del conocimiento y crecimiento en la Pyme manufacturera de Aguascalientes (México)}

\section{Gestion de la connaissance et croissance chez la PME manufacturière $\mathrm{d}^{-}$ Aguascalientes (Mexique)}

\section{Abstract}

Within a constantly changing market and an uncertain environment for business, knowledge has become one of the valued intangible resources appreciated by firms. For this, more and more firms are applying managerial strategies to promote the development of knowledge, both tacit and implicit, in such a way that allows them to achieve expected success. Also, an important number of companies, mainly small and mediumsized enterprises, are getting involved with knowledge management as part of their daily activities, seeking to improve higher growth. Thus, the value of knowledge is increasing, to such a measure that firms are hiring the most brilliant minds in each specialty. In this sense, this paper aims to analyze the relationship between knowledge management and growth, starting from a sample of 125 SMEs from the State of Aguascalientes (Mexico). The results obtained show that knowledge management has positive and significant effects on the growth of SMEs, which can constitute a competitive advantage.

Keywords: knowledge, knowledge management, growth, SMEs.

\section{Gestion de la connaissance et croissance chez la PME manufacturière $d^{\prime}$ Aguascalientes (Mexique)}

\section{Gestión del conocimiento y crecimiento en la Pyme manufacturera de Aguascalientes (México)}

\section{Knowledge management and growth in manufacturing SMEs from Aguascalientes (Mexico)}

\section{Résumée}

Dans un marché à changements constants et une ambiance $d^{\prime}$ 'incertitude dans les affaires, la connaissance est devenue une des ressources intangibles la plus valorisée et appréciée des entreprises. Voilà pourquoi, les entreprises appliquant des stratégies entrepreneuriales sont de plus en plus nombreuses, pour promouvoir le développement de la connaissance, aussi bien tacite qu'implicite, permettant de telle façon réussir au succès prévu. Pour ça, un nombre important d'entreprises, surtout petites et moyennes (PME), incorporent la gestion de la connaissance comme partie de leurs activités quotidiennes, avec l'objectif d'améliorer leur niveau de croissance. La valeur de la connaissance augmente chaque fois de plus, à tel point que les entreprises embauchent les pensées les plus brillantes de chaque spécialité. Le but de cette étude est d'analyser la relation qui existe entre la gestion de la connaissance et la croissance, en utilisant un échantillon de 125 PME de l'état d'Aguascalientes (Mexique). Les résultats obtenus montrent que la gestion de la connaissance a des effets positifs et significatifs dans la croissance de la PME, ce qui peut constituer un avantage compétitif.

Mots clef: connaissance, gestion de la connaissance, croissance, PME. 


\section{Gestión del conocimiento y crecimiento en la Pyme manufacturera de Aguascalientes (México)}

\section{Introducción}

El concepto de gestión del conocimiento (KM) ha sido ampliamente analizado y discutido en la literatura, y con frecuencia se considera como un constructo multidimensional. Durante la década de 1990 , el concepto de la KM se utilizó para describir a las aplicaciones computacionales y a las tecnologías de la información y comunicación (Wilson, 2002). En referencia a este concepto, Wilson (2002) consideró que la KM tiene un significado diferente en las organizaciones, y cada una de las empresas trata de implementarlo de acuerdo con su manera particular de entenderlo

Wilson (2002) también argumentó que realmente son muy pocas las empresas que implementan en su organización a la KM, y prueba de ello es el estudio realizado por la empresa Bain en el año 2000 (Bain Company, 2001), en el que se establece que solo el $35 \%$ de las 451 empresas analizadas habían utilizado a la KM en su organización, y habían logrado un nivel de satisfacción de 3.5 en una escala tipo Likert de 5 puntos. Además, Wilson (2002) concluyó que la KM ocupó el lugar 19 de los 25 factores más importantes de la gestión de los negocios, comparado con el 70\% de las empresas que utilizan el benchmarking o el $80 \%$ que utilizan la planeación estratégica.

Las propuestas realizadas por Wilson (2002) han desatado una fuerte polémica entre los distintos investigadores especializados en el campo de la KM, a tal extremo de que existe en la actual literatura un número considerable de trabajos de investigación que tienen posiciones encontradas, unos de ellos se muestran a favor y otros más en contra de estas argumentaciones (Kruger y Johnson, 2009). Sin embargo, existe un consenso entre un número importante de investigadores que han negado la perspectiva de información de la KM. Este grupo de autores consideran que más bien la KM puede ser vista como una perspectiva de estrategia, gestión e innovación en la organización (Sveiby, 1990, 1997; Nonaka y Takeuchi, 1995; von Krogh, Nonaka y Aben, 2001).

Es común que en la literatura se le relacione a la KM con el aprendizaje de la empresa, entendido este último como el proceso por el cual la organización aprende de sus mejores prácticas y evita repetir los errores, porque el aprendizaje se genera por medio del enfoque que tenga la organización en sus activos de conocimiento, crecimiento y desarrollo de los canales por los que fluye el conocimiento. Por lo tanto, el éxito del aprendizaje de la organización estriba en la transformación del conocimiento del capital humano (conocimiento tácito que tienen las personas) en conocimiento del capital estructural (conocimiento explícito) (Sharma, Siddiqui, Sharma, Sing, Kumar, Kaushal y Banerjee, 2007).

Por otro lado, el proceso del ciclo de vida del conocimiento se desarrolla en la organización a un ritmo muy vertiginoso porque comúnmente el conocimiento se desenvuelve por medio del conocimiento explícito, que es codificable, y el conocimiento implícito o tácito, que no es codificable pero se encuentra en la experiencia y habilidades de los trabajadores (Birkinshaw y Sheehan, 2002). Por lo tanto, el conocimiento explícito puede ser articulado en la organización sobre todo por medios escritos tales como las normas, valores y el entrenamiento de los empleados, mientras que el conocimiento implícito es aquel que se manifiesta por medio de la experiencia y habilidades que poseen los trabajadores de la organización (Sharma et al., 2007).

En este mismo contexto, Nonaka y Takeuchi (1995) analizaron con mayor profundidad estos dos tipos de conocimiento, siendo los primeros autores en popularizar estos términos, y concluyeron en su estudio que ambos tipos de conocimientos son creados, capturados, organizados, renovados, buscados y transferidos para su reúso. Asimismo, Hansen, Nohria y Tierney (1999) consideraron que mientras el conocimiento explícito puede ser compartido tanto por los trabajadores como por los empleados, a lo que ellos llamaron codificación, el conocimiento que tiene mayor valor para las empresas es aquel que se genera con el intercambio de conocimiento tácito, a lo que ellos llamaron personalización.

Otros investigadores han encontrado nuevas formas de intercambio de conocimiento al interior de la organización; por ejemplo Choo y Brontis (2002), consideraron que existe un dilema en 
encontrar un balance entre la exploración del conocimiento, que es el descubrimiento y creación de conocimiento que impacte en las actividades esenciales de las empresas y en la competencia, y la explotación del conocimiento, que es la existencia del conocimiento que permite a los negocios adquirir o mejorar sus ventajas competitivas, porque este balance es esencial para asimilar todo el conocimiento intensivo que se genera en las organizaciones.

Igualmente, Nonaka y Takeuchi (1995) concluyeron que se requiere de un espacio de trabajo adecuado, que pueda facilitar el intercambio de conocimiento en la organización, mientras que Zack (1999) consideró que la no alineación de las estrategias empresariales y de conocimiento, puede ser una de las causas esenciales de que la organización tenga un pobre desempeño. Gupta y Govindarajan (2000) realizaron una extensa investigación sobre los flujos de conocimiento en las empresas multinacionales, y llegaron a la conclusión de que no alinear los flujos de conocimiento de los recursos disponibles y el público objetivo, puede generar ineficiencia en las organizaciones. Por lo tanto, la motivación esencial para generar una plataforma de la KM en cualquier organización, debe ser la realización de que el conocimiento es un valor esencial que puede ser compartido y reusado cuando se presenten las oportunidades de negocio (Sharma et al., 2007).

Respecto de la aplicación de la KM en la Pyme, son pocos los trabajos publicados en la literatura que presentan evidencia empírica de la relación existente entre la KM y el crecimiento. Entre los estudios más destacados se encuentra el presentado por Beijerse (2000), quien a través de una investigación realizada a 12 Pymes de Holanda, encontró 79 diferentes actividades o procesos de $\mathrm{KM}$, pero lo más sorprendente del estudio es que todas las actividades de KM estaban relacionadas con la gestión estratégica y con una cultura organizacional abierta y positiva.

Del mismo modo, Lim y Klobas (2000) estudiaron a las Pymes de Australia y Singapur y encontraron que las necesidades y cambios en la KM son demasiado similares a las que tienen las grandes empresas de esos dos países. Asimismo, estos investigadores también identificaron que diversos procesos de gestión del conocimiento de las grandes empresas, pueden ser aplicados sin ningún problema en las Pymes porque comúnmente es muy sencilla la adquisición de conocimiento tácito en pequeños ambientes formales.
Otros estudios como los realizados por Gustavson y Harung (1994) y Choueke y Armstrong (1998), encontraron que un ambiente colectivo y una transferencia de experiencias entre los trabajadores de las Pymes, tienen un fuerte impacto tanto en el aprendizaje de la organización y en la habilidad para cambiar, como en las ventajas competitivas. Por lo tanto, las Pymes pueden estar en condiciones para incrementar significativamente su crecimiento y sus ventajas competitivas a través de una KM mejor gestionada y sistemática.

Por su parte, Penn, Ang'wa, Forster, Heydon y Richardson (1998) y Matlay (2000) analizaron las Pymes en Inglaterra, y concluyeron que la estrategia de aprendizaje y la orientación al conocimiento, son dos factores esenciales que pueden ayudar a las Pymes a sobrevivir y crecer a largo plazo. Asimismo, consideraron que a pesar de que el aprendizaje ocurre en la mayoría de las Pymes, solo unas cuantas empresas tiene la capacidad de gestionar estratégicamente nuevo conocimiento para mantener e incrementar sus ventajas competitivas. Por lo tanto, los resultados obtenidos de estos dos estudios, muestran que la estrategia de adquisición de conocimiento es mucho más importante para el crecimiento de la Pyme a largo plazo que los factores medioambientales que la rodean (Salojärvi, Furu y Sveiby, 2005).

Existe diversas clasificaciones de la KM en la actual literatura del campo de la gestión del conocimiento, pero la más reciente es la presentada por Bozbura (2007), quien realizó una extensa revisión de la literatura y llegó a la conclusión de que la KM se puede clasificar en cuatro dimensiones esenciales: 1) entrenamiento de los empleados; 2) políticas y estrategias de la KM; 3) creación y adquisición de conocimiento externo; 4) efectos de la cultura organizacional.

En este sentido, Bozbura (2007) consideró que el capital humano es el activo de mayor importancia para cualquier organización, porque es precisamente éste el generador de nuevo conocimiento, y es tan importante que cada vez son más las Pymes que invierten más recursos económicos en un entrenamiento para incrementar sus capacidades, experiencia y conocimiento (Ulrich, 1997). Por lo tanto el entrenamiento de los empleados tiene una relación directa con la KM de la organización (Becker, Huselid y Ulrich, 2001). Así, ahora se puede plantear la siguiente hipótesis: 
H1: A mayor nivel de entrenamiento de los empleados, mayor nivel de gestión del conocimiento

La misión, visión, estrategias y los procesos de gestión de las Pymes pueden ser factores esenciales que mejoren las habilidades y fortalezcan la creatividad de los empleados, por lo cual estos elementos pueden ser definidos como políticas y estrategias que afectan directamente a la KM de las organizaciones (Bozbura, 2007). Por ello, ahora se puede plantear la siguiente hipótesis:

H2: A mayor nivel de aplicación de políticas $y$ estrategias de KM, mayor nivel de gestión del conocimiento

La creación y adquisición de conocimiento es otro de los elementos esenciales para la KM, porque también el conocimiento se puede obtener por medio de las relaciones de las Pymes con sus clientes, proveedores, competencia, sociedad e instituciones oficiales (Bozbura, 2007). Por lo tanto, en este momento se puede plantear otra hipótesis:

H3: A mayor nivel de creación y adquisición de conocimiento, mayor nivel de gestión del conocimiento

Para que los empleados puedan compartir el conocimiento o la información que poseen al interior de las Pymes, se requiere del diseño y uso de canales de comunicación eficientes lo que permitirá que la organización cuente con una eficiente KM. En este sentido, las condiciones de trabajo, el trabajo en equipo, los canales para compartir el conocimiento, la creación de un ambiente favorable para la libre expresión de las opiniones pueden ser definidas como los efectos directos de la cultura organizacional en la KM (Zack, 1999; Bontis, 2000; Buckley y Carter, 2000; OECD, 2003; Bozbura, 2004; 2007). Así, ahora se puede plantear la siguiente hipótesis:

H4: A mayor nivel de efecto de la cultura organizacional, mayor nivel de gestión del conocimiento

Respecto del crecimiento en las Pymes, este por lo general se considera como una de las mediciones del desempeño de los negocios más importantes (Storey, 1994; Goold, 1996). A pesar de que el crecimiento es una medida esencial de la evaluación de la reserva del mercado, comúnmente no se reconoce como una medida de las expectativas de crecimiento para la capitalización del mercado. Sin embargo, al crecimiento se le considera como uno de los criterios elementales cuando se evalúa el éxito de las Pymes, en especial el crecimiento de las ventas (Watson, Hogarth-Scott y Wilson, 1998; Laurence, 2001; 0־Gorman, 2001), y es el factor esencial para medir la prosperidad en las sociedades modernas (Charan y Tichy, 2000).

Mouritsen (1998, p. 461) consideró que “el crecimiento y la creación del valor financiero no puede considerarse solamente como un posible objetivo para la gestión a futuro de la organización, en la actualidad esto sucede con regularidad". Por lo tanto, mantener una estabilidad del crecimiento en la Pyme puede resultar muy difícil en el largo plazo (Goold, 1996). Sin embargo, el crecimiento sostenido (por ejemplo, el crecimiento en el largo plazo se considera como negocios maduros) se estima con regularidad en la literatura como uno de los indicadores esenciales del éxito de las organizaciones (O’Gorman, 2001).

Salojärvi et al. (2005) propusieron tres razones fundamentales para considerar al crecimiento como una de las variables más importantes para medir el desempeño de las Pymes. Primero, el crecimiento de las ventas en varios estudios, muestran que la mayoría de los gerentes de las Pymes creen que el crecimiento es muy importante (Penn et al., 1998), y que el crecimiento como medida está más estrechamente relacionado con las metas estratégicas de las organizaciones que el beneficio como medida (Hudson, Smart y Bourne, 2001). Asimismo, Smallborne, Leigh y North (1995) piensan que una de las características más importantes que distinguen al desempeño de las Pymes de otras organizaciones, es precisamente su compromiso con el crecimiento. Segundo, el crecimiento en las Pymes ha sido identificado en la mayoría de las sociedades, como una de las variables de mayor importancia de las estrategias económicas, para lograr aumentar el número de trabajadores y mejorar la estabilidad de la organización (Carson, Cromie, McGowan y Hill, 1995; Hodgetts y Kuratko, 1995; Holmlund y Kock, 1998). Tercero, el crecimiento es probablemente el indicador de mayor fiabilidad para los gerentes o dueños de las Pymes, que los indicadores relacionados con la ganancia.

Por otro lado, no existe en la literatura una teoría en particular que explique el crecimiento en las Pymes, por lo cual se debe trabajar en el futuro en el desarrollo de una teoría que explique esta importante variable (Gibb y Davies, 1990). 
Asimismo, en estudios recientes se ha hecho énfasis en que las Pymes deben aprender habilidades, tener una cultura abierta y desarrollar un liderazgo para mejorar su nivel de crecimiento (Smallborne et al., 1995; Smith, 1998; Watson et al., 1998; Choueke y Armstrong, 2000; Zhang, 2000; Weinzimmer, 2000; Morrison y BerginSeers, 2002).

Finalmente, un cambio esencial en el crecimiento de las Pymes es sin lugar a dudas la flexibilidad y el nivel de innovación, mientras que al mismo tiempo tiene que introducir procesos sistemáticos, porque estos cambios son fundamentales para lograr el éxito de las organizaciones (Hambrick y Crozier, 1985), y la aplicación de una estrategia de gestión del conocimiento puede incrementar el nivel de su crecimiento (Salojärvi et al., 2005; Kruger y Johnson, 2009). Así, bajo este contexto y considerando la información antes presentada, se puede plantear ahora la última hipótesis.

H5: A mayor nivel de gestión del conocimiento, mayor nivel de crecimiento

\section{Metodología}

Para contrastar las hipótesis planteadas en el modelo teórico, se aplicó un modelo de ecuaciones estructurales (MEC) utilizando el software EQS 6.1 (Bentler, 2005; Byrne, 2006; Brown, 2006), donde la validez nomológica del modelo teórico fue comparada con la del modelo ajustado, a través del análisis del desempeño de la prueba Chi cuadrado (c2). Asimismo, se aplicó un estudio empírico en la Pyme manufacturera del estado de Aguascalientes (México), considerando para ello el Directorio del Sistema de Información Empresarial de México del año 2009 para el estado de Aguascalientes, el cual tenía registradas hasta el 30 de julio del mismo año 130 empresas de manufactura que tenían entre 20 y 250 empleados.

Considerando que la población objeto de estudio era muy pequeña (130 Pymes), se tomó la decisión de aplicar una entrevista personal a los gerentes generales $\mathrm{y} / \mathrm{o}$ propietarios de todas las empresas que reunían las características deseadas, recibiendo un total de 125 encuestas y obteniendo una tasa de respuesta del $96 \%$. Asimismo, las encuestas se aplicaron entre los meses de septiembre a diciembre de 2009. La Tabla 1 resume los aspectos más importantes de la investigación realizada.

\begin{tabular}{|c|c|}
\hline Características & Investigación \\
\hline Población & $\begin{array}{l}130 \text { pequeñas y medianas } \\
\text { empresas }\end{array}$ \\
\hline Área Geográfica & $\begin{array}{l}\text { Estado de Aguascalientes } \\
\text { (México) }\end{array}$ \\
\hline Muestra & Pymes de 20 a 250 empleados \\
\hline $\begin{array}{l}\text { Método de recolección de } \\
\text { la información }\end{array}$ & $\begin{array}{l}\text { Entrevistas personales a los } \\
\text { gerentes }\end{array}$ \\
\hline Método de muestreo & Muestreo aleatorio simple \\
\hline Tamaño de la muestra & 125 Pymes \\
\hline Error del muestreo & $\begin{array}{l}+1 \% \text { error, nivel de } \\
\text { confiabilidad del } 99 \% \\
(p=q=0.5)\end{array}$ \\
\hline Trabajo de campo & Septiembre a diciembre de 2009 \\
\hline
\end{tabular}

\subsection{Construcción del instrumento de medición}

El instrumento de medición se diseñó considerando las medidas de la KM más actuales encontradas en la literatura. Por lo tanto, para medir a la KM se tomaron en cuenta las cuatro dimensiones propuestas por Bozbura (2007). Así, el entrenamiento de los empleados se medió utilizando una escala de 5 puntos y adaptada de Bontis (2000) y OECD (2003). La escala para medir las políticas y estrategias de la KM fue una escala de 13 puntos y adaptada de Bozbura $(2004,2007)$. La escala para medir la creación y adquisición de conocimiento externo fue medida por medio de una escala de 5 puntos y adaptada de OECD (2003) y de Bozbura (2007). Por último, la escala para medir los efectos de la cultura organizacional en la KM fue una escala de 4 ítems y adaptada también de OECD (2003) y de Bozbura (2007). Todos estos puntos se midieron por medio de una escala tipo Likert de 5 puntos con $1=$ Total desacuerdo a $5=$ Total acuerdo como límites.

Por otro lado, el crecimiento se midió a través de las ventas realizadas por las Pymes en el 2010 (Autio y Lumme, 1998; Ballow et al., 2004; Salojärvi et al., 2005; Linder, 2006; Carneiro, 2007; Kruger y Johnson, 2009). Asimismo, para estimar el potencial de crecimiento que pueden tener las Pymes, por lo general se considera una evaluación cualitativa de los gerentes siendo las ventas el principal indicador para su medición (Autio y Lumme, 1998).

\subsection{Fiabilidad y validez}

Para evaluar la fiabilidad y validez de las cuatro dimensiones de la KM, se utilizó por una parte el análisis factorial confirmatorio (AFC) 
(Bentler, 2005; Brown, 2006; Byrne, 2006) y, por otra parte el coeficiente alfa de Cronbach y el índice de fiabilidad compuesta (IFC) (Bagozzi y $Y i, 1988)$. También se utilizaron otros métodos de estimación de los estadísticos del modelo teórico, cuando se asume que no existe normalidad de los datos (Chou, Bentler y Satorra, 1991; Hu, Bentler y Kano, 1992), y se consideraron las recomendaciones de Satorra y Bentler (1988) de la utilización de los estadísticos robustos para mejorar el ajuste estadístico del modelo.

En cuanto a los índices de ajuste que se utilizaron en el modelo teórico, estos fueron el $\mathrm{NFI}, \mathrm{NNFI}, \mathrm{CFI}$ y RMSEA (Bentler y Bonnet, 1980; Byrne, 1989; Bentler, 1990; Hair, Anderson, Tatham y Black, 1995; Chau, 1997; Heck, 1998). Por lo tanto, si el valor de los tres primeros índices se encuentra entre 0.8 y 0.89 se considera que el modelo teórico tiene un ajuste razonable (Segars y Grover, 1993). Sin embargo si este valor es igual o superior a 0.9 se considera que el modelo teórico tiene un buen ajuste (Jöreskog y Sörbom, 1986; Byrne, 1989; Papke-Shields, Malhotra y Grover, 2002); por el contrario, si el valor del RMSEA es inferior a 0.08 se considera como aceptable (Jöreskog y Sörbom, 1986, Hair et al., 1995).

La Tabla 2 muestra los resultados obtenidos del AFC y sugieren, por un lado, que el modelo tiene un buen ajuste $\left(\mathrm{S}-\mathrm{BX}^{2}=110.888 ; \mathrm{df}=49 ; \mathrm{p}\right.$
$=0.000 ; \mathrm{NFI}=0.904 ; \mathrm{NNFI}=0.927 ; \mathrm{CFI}=0.929 ; \mathrm{y}$ RMSEA $=0.074) y$, por el otro, que existe una alta consistencia interna de los constructos, porque el valor del alfa de Cronbach y el IFC es superior a 0.7 (Nunnally y Bernstein, 1994), el tamaño de todas las cargas factoriales estandarizadas de las variables son significativas ( $p<0.001)$ y superiores a 0.6 (Bagozzi y Yi, 1988), lo que proporciona una evidencia de fiabilidad del modelo teórico y justifica la fiabilidad interna de las escalas (Nunally y Bernstein, 1994; Hair et al., 1995). Por último, el índice de la varianza extraída (IVE) es superior a 0.5 como lo recomienda Fornell y Larcker (1981).

En la Tabla 3 se pueden apreciar los resultados obtenidos en lo referente a la validez discriminante del modelo teórico. En primer lugar, por encima de la diagonal se presenta la prueba del intervalo de confianza (Anderson y Gerbing, 1988), que establece que con un intervalo del $95 \%$ de confidencialidad ninguno de los elementos individuales de los factores latentes de la matriz de correlación contiene el valor 1.o. En segundo lugar, por debajo de la diagonal se presenta la prueba de la varianza extraída (Fornell y Larcker, 1981), que establece que la varianza extraída (la correlación al cuadrado) entre cada par de factores debe ser inferior que su correspondiente IVE. Por lo tanto, se puede concluir que las distintas mediciones realizadas en este modelo demuestran suficiente evidencia de fiabilidad y validez convergente y discriminante.

\begin{tabular}{|c|c|c|c|c|c|c|}
\hline Variable & Indicador & $\begin{array}{c}\text { Carga } \\
\text { Factorial }\end{array}$ & $\begin{array}{c}\text { Valor t } \\
\text { Robusto }\end{array}$ & $\begin{array}{c}\text { Alfa de } \\
\text { Cronbach }\end{array}$ & IFC & IVE \\
\hline Entrenamiento de los Empleados & $\begin{array}{l}\mathrm{BFT}_{1} \\
\mathrm{BFT}_{3} \\
\mathrm{BFT}_{4}\end{array}$ & $\begin{array}{l}0.798^{\star \star \star} \\
0.840^{\star \star \star} \\
0.763^{\star \star \star}\end{array}$ & $\begin{array}{l}1.000^{a} \\
11,314 \\
8,863\end{array}$ & 0,842 & 0,843 & 0,642 \\
\hline Políticas y Estrategias & $\begin{array}{l}\text { BPE2 } \\
\text { BPE3 }_{3} \\
\text { BPE4 }\end{array}$ & $\begin{array}{l}0.825^{\star \star \star} \\
0.800^{\star \star \star} \\
0.794^{\star \star \star}\end{array}$ & $\begin{array}{c}1.000^{a} \\
9,45^{8} \\
9,392\end{array}$ & 0,846 & 0,848 & 0,65 \\
\hline Adquisición de Conocimiento Externo & $\begin{array}{l}\mathrm{BKO}_{1} \\
\mathrm{BKO}_{2} \\
\mathrm{BKO}_{3} \\
\mathrm{BKO}_{5}\end{array}$ & $\begin{array}{l}0.719^{\star \star \star} \\
0.779^{\star \star \star} \\
0.793^{\star \star \star} \\
0.715^{\star \star \star}\end{array}$ & $\begin{array}{c}1.000^{a} \\
9,804 \\
7,938 \\
7,791\end{array}$ & 0,833 & 0,839 & 0,566 \\
\hline Efectos de la Cultura Organizacional & $\begin{array}{l}\mathrm{BOC}_{1} \\
\mathrm{BOC}_{2} \\
\mathrm{BOC}_{4}\end{array}$ & $\begin{array}{l}0.817^{\star \star \star} \\
0.831^{\star \star \star} \\
0.704^{\star \star \star}\end{array}$ & $\begin{array}{c}1.000^{a} \\
11,510 \\
8,150\end{array}$ & 0,827 & 0,829 & 0,618 \\
\hline
\end{tabular}


demuestra la importancia que tiene la gestión del conocimiento en las organizaciones, principalmente en las Pymes.

Los resultados empíricos encontrados en este estudio también indican que si las Pymes quieren mejorar su nivel de crecimiento, tendrán que implementar entre otros factores, estrategias empresariales tendientes a gestionar adecuadamente tanto el conocimiento tácito como el implícito existente en los trabajadores de la organización. Por lo tanto, los gerentes tendrán que realizar una serie de actividades que generen un ambiente favorable para que los trabajadores de la organización puedan compartir conocimientos, habilidades y experiencias con sus demás compañeros.

Para que las Pymes puedan mejorar la gestión del conocimiento se requiere de la implementación de diversas actividades tales como un adecuado entrenamiento de los empleados en aquellas actividades esenciales de la organización, el diseño y aplicación de políticas y estrategias de gestión del conocimiento, la adquisición de conocimiento externo, sobre todo de las instituciones públicas de educación superior y centros de investigación especializados en las Pymes y, mejorar la cultura organizacional para crear espacios propicios para el intercambio de conocimiento y experiencia entre los empleados de la organización.

Este estudio empírico presenta una serie de implicaciones tanto para las Pymes como para los gerentes; entre las más importantes se encuentra, aquella donde los gerentes tienen que comprometerse más en desarrollar e implementar estrategias de gestión del conocimiento, tanto al interior como al exterior de la organización, con el objetivo de mejorar de forma significativa el nivel de crecimiento de la empresa. Por lo tanto, los gerentes deben involucrar a todo el personal de la organización, tanto en el diseño como en la implementación de las estrategias, de tal manera que se genere un ambiente propicio que facilite el intercambio de conocimiento y experiencias.

Otra implicación es la referente al crecimiento de las ventas de la organización, donde los gerentes deberán implementar estrategias empresariales tendientes a aumentar el nivel de las ventas y buscar mecanismos que propicien que las ventas de la empresa no disminuyan, porque se vería seriamente afectado el nivel de crecimiento de la Pyme. Por lo tanto, los gerentes tendrán que rediseñar las estrategias de la organización, para adecuarlas o alinearlas a las de sus clientes y proveedores, con la única finalidad de que sus ventas no se vean seriamente afectadas por una disminución.

Otra implicación esencial es la referente a la generación y adquisición de información relevante de la gestión del conocimiento y del mercado, de tal manera que permita, por una parte, una solución oportuna a los posibles problemas de gestión del conocimiento que se puedan presentar tanto al interior como al exterior de la organización. Por otra parte, la información del mercado debe propiciar la mejor toma de decisiones, con respecto al potencial de las ventas y la rentabilidad del mercado en el que participa la Pyme, para que su nivel de ventas no se vea reducido por una mala decisión.

Por otro lado, este estudio presenta una serie de limitaciones que es preciso aclarar. Primero se encuentra la limitante de la muestra, porque solo se consideraron a las pequeñas y medianas empresas de 20 a 250 trabajadores del estado de Aguascalientes (México), con lo cual se dejó fuera del estudio a las microempresas (de 1 a 10 trabajadores), y a una parte considerable de las pequeñas empresas (de 11 a 19 trabajadores), que en conjunto representan un poco más del $70 \%$ de la población total, con lo cual para estudios futuros sería necesario considerar tanto a las empresas de menor tamaño como a las Pymes de otros estados para ver el comportamiento de estas variables.

En segundo lugar, está la limitante de la obtención de la información, pues solo se consideró en la encuesta una parte de la información total de la gestión del conocimiento. Asimismo, se dificultó demasiado la obtención de la información, porque en su mayoría las empresas consideraron que la información solicitada era muy confidencial y no estaban dispuestas a proporcionar toda la información solicitada, por lo cual se pidió una entrevista con el gerente general de la Pyme para explicarle el alcance de la investigación y que los datos serían tratados en general y no en particular.

Por último, otra limitante es que el instrumento para medir la gestión del conocimiento y el crecimiento se aplicó solo a los gerentes generales y/o propietarios de las Pymes, por lo cual se asume que tienen un amplio conocimiento de los cuatro factores utilizados para medir esta. Sería adecuado que en futuros estudios se aplicara esta encuesta tanto a los empleados como a los clientes de la organización, para recabar información desde otra óptica y correlacionarla con la información proporcionada por los gerentes. 
Finalmente, en la escala de la gestión del conocimiento se consideraron solo cuatro dimensiones, y para medir el crecimiento se consideró el crecimiento de las ventas, por lo cual sería de suma importancia que en estudios futuros se tuvieran en cuenta otras medidas del crecimiento más cuantitativas. Por lo tanto, cabría preguntarse en estos momentos ¿qué pasaría con el crecimiento de la organización si se considerara otra variable más cuantitativa? 0 bien ¿qué pasaría con la gestión del conocimiento si se consideraran otros factores para su medición? Estas y otras preguntas que puedan desprenderse de este estudio empírico, se pueden contestar en estudios futuros.

\section{Referencias}

Anderson, J. \& Gerbing, D. (1988). Structural equation modeling in practice: a review and recommended two-step approach. Psychological Bulletin 13 (pp. 411-423).

Auttio, E. \& Lumme, A. (1998). Does the innovator role affect the perceived potential for growth? Analysis of four types of new, technology-based firms. Technology Analysis \& Strategic Management, 10 (1), (pp. 41-54).

Bagozzi, R.P. \& Yi, Y. (1988). On the evaluation of structural equation models. Journal of the Academy of Marketing Science, 16 (1), (pp. 74-94).

Ballow, J.J., McCarthy. B. \& Molnar, M.J. (2004). New concepts in value-based management: trs mapping and total economic profit. Wellesley, USA: Accenture Institute for High Performance Business.

Becker, B.E., Huselid, M.A. \& Ulrich, D. (2001). The $H R$ scorecard. Boston, USA: Harvard Business School Press.

Beijerse, R.P. (2000). Knowledge management in small and medium-sized companies: knowledge management for entrepreneurs. Journal of Knowledge Management, 4 (2), (pp. 162-179).

Bentler, P.M. (1990). Comparative fit indexes in structural models. Psychological Bulletin, 107 (2), (pp. 238-246).

Bentler, P.M. (2005). EQS 6 structural equations program manual. Encino, USA: Multivariate Software.
Bentler, P.M. \& Bonnet, D. (1980). Significance tests and goodness of fit in analysis of covariance structures. Psychological Bulletin 88 (pp. 588606).

Birkinshaw, J. \& Sheehan, T. (2002). Managing the knowledge life cycle. MIT Sloan Management Review, 44 (1) (pp.75-83).

Bontis, N. (2000). Intellectual capital and business performance in Malaysian industries. Journal of Intellectual Capital, 1 (1), (pp. 85-100).

Bozbura, F.T. (2004). Measurement and application of intellectual capital in Turkey. The Learning Organization. An International Journal, 11 (4/5), (pp. 357-367).

Bozbura, F.T. (2007). Knowledge management practices in Turkish SMEs. Journal of Enterprise Information Management, 20 (2), (pp. 209-221).

Brown, T. (2006). Confirmatory factor analysis for applied research. New York, USA: The Guilford Press.

Buckley, P.J. \& Carter, M.J. (2000). Knowledge management in global technology markets. Long Range Planning 33, (pp. 55-71).

Byrne, B.M. (1989). A primer of LISREL: basic applications and programming for confirmatory factor analysis analytic models. New York, USA: Springer.

Byrne, B.M. (2006). Structural equation modeling with EQS, basic concepts, applications, and programming. (2 ed.). London, UK:. LEA Publishers.

Carneiro, A. (2007). What is required for growth? Business Strategy Series, 8 (1), (pp. 51-57).

Carson, D.J., Cromie, S., McGowan, P. \& Hill, J. (1995). Marketing and entrepreneurship in SMEs: an innovative approach. London, UK: Prentice-Hall.

Charan, R. y Tichy, N.M. (2000). Every business is a growth business. New York, USA: Three Rivers Press.

Chau, P. (1997). Reexamining a model for evaluating information center success using a structural equation modeling approach. Decision Sciences, 28 (2), (pp. 309-334). 
Choo, C.W. \& Brontis, N. (2002). The strategic management of intellectual capital and organizational knowledge. Oxford, UK: University Press.

Chou, C.P., Bentler, P.M. \& Satorra, A. (1991). Scaled test statistics and robust standard errors for nonnormal data in covariance structure analysis. British Journal of Mathematical and Statistical Psychology 44, (pp. 347-357).

Choueke, R. \& Armstrong, R. (1998). The learning organization in small and medium-sized enterprises: a destination or a journey. International Journal of Entrepreneurial Behaviour \& Research, 4 (2), (pp. 129-140).

Choueke, R. \& Armstrong, R. (2000). Culture: a missing perspective on small and medium-sized enterprise development. International Journal of Entrepreneurial Behaviour \& Research, 6 (4), (pp. 227-238).

Fornell, C. \& Larcker, D. (1981). Evaluating structural equation models with unobservable variables and measurement error. Journal of Marketing Research, 18, (pp. 39-50).

Gibb, A. \& Davies, L. (1990). In pursuit of framework for the development of growth models of the small business. International Small Business Journal, 9 (1), (pp. 15-31).

Goold, M. (1996). The growth imperative. Long Range Planning, 32 (1), (pp. 127-129).

Gupta, A.K. \& Govindarajan, V. (2000). Knowledge flows within multinational corporations. Strategic Management Journal, 21, (pp. 473-496).

Gustavson, B. \& Harung, H.S. (1994). Organizational learning based on transforming collective consciousness. The Learning Organizations, 1 (1), (pp. 33-40).

Hair, J.F., Anderson, R.E., Tatham, R.L. \& Black, W.C. (1995). Multivariate data analysis with readings. New York, USA: Prentice-Hall.

Hambrick, D.C. \& Crozier, L.M. (1985). Stumblers and stars in the management of rapid growth. Journal of Business Venturing, 1 (1), (pp. 31-45).

Hansen, M., Nohria, N. y Tierney, T. (1999/04). What's your strategy for managing knowledge., Harvard Business Review, (pp. 106-116).
Hatcher, L. (1994). A step by step approach to using the SAS system for factor analysis and structural equation modeling. Cary, USA: SAS Institute Inc.

Heck, R.H. (1998). Factor analysis: exploratory and confirmatory approaches, en Marcoulides, G.A. (Ed.), Modern Methods for Business Research. Mahwah. (pp. 177-215). USA: Lawrence Erlbaum Associates.

Hodgetts, R.M. \& Kuratko, D.R. (1995). Effective small business management ( 5 ed.) Fort Worth, USA: The Dryden Press.

Holmlund, M. \& Kock, S. (1998). Relationships and the internationalization of finnish small and medium-sized companies. International Small Business Journal, 16 (64), (pp. 46-63).

Hu, L.T., Bentler, P.M. \& Kano, Y. (1992). Can test statistics in covariance structure analysis be trusted? Psychological Bulletin 112, (pp. 351-362).

Hudson, M., Smart, A. \& Bourne, M. (2001). Theory and practice in SME performance measurement systems. International Journal of Operations \& Production Management 21 (8), (pp.1096-1115).

Jöreskog, K.G. \& Sörbom, D. (1986). LISREL VI: analysis of linear structural relationships by maximum likelihood, instrumental variables and square methods. Moorsville, USA: Scientific Software.

Kruger, C.J. \& Johnson, R.D. (2009). Assessment of knowledge management growth: a South Africa perspective. Aslib Proceedings, New Information Perspectives, 61 (6), (pp. 542-564).

Laurence, G. (2001). Fast growth: how to attain it, how to sustain it. Chicago, USA: Kaplan Professional Company.

Lim, D. \& Klobas, J. (2000). Knowledge management in small enterprises. The Electronic Library 18 (6), (pp. 420-432).

Linder, J.C. (2006). Does innovation drive profitable growth? New metrics for a complete picture. Journal of Business Strategy 27 (5), (pp. 38-44).

Matlay, H. (2000). Organizational learning in small learning organizations: an empirical overview. Educational \& Training, 4 (5), (pp. 202-210). 
Morrison, A. \& Bergin-Seers, S. (2002). Pro-growth small businesses: learning architecture. Journal of Management Development 21 (5), (pp. 388400).

Mouritsen, J.(1998). Driving growth: economic value added versus intellectual capital. Management Accounting Research 9 (4), (pp. 461-482).

Nonaka, I. \& Takeuchi, H. (1995). The knowledgecreating company. New York, USA: Oxford University Press.

Nunnally, J.C. \& Bernstein, I.H. (1994). Psychometric theory (3 ed.). New York, USA: McGraw-Hill.

O'Gorman, C. (2001). The sustainability of growth in small and medium-sized enterprises. International Journal of Entrepreneurial Behaviour \& Research, 7 (2), (pp. 60-75).

OECD. (2003). Measuring knowledge management in the business sector. Ottawa, Canadá: Organization for Economic Co-operation and Development (OECD), Minister of Industry.

Papke-Shields, K.E., Malhotra, M.J. \& Grover, V. (2002). Strategic manufacturing planning systems and their linkage to planning system success. Decision Science, 13 (1), (pp. 1-30).

Penn, D.W., Ang'wa, W., Forster, R., Heydon, G. y Richardson, J.S. (1998). Learning in smaller organizations. The Learning Organization, 5 (3), (pp. 128-137).

Rigby, D.K. (2001). Management Tools. Boston, USA: Bain and Company.

Salojärvi, S., Furu, P. \& Sveiby, K.E. (2005). Knowledge management and growth in finnish SMEs. Journal of Knowledge Management, 9 (2), (pp. 103-122).

Satorra, A. \& Bentler, P.M. (1988). Scaling corrections for chi square statistics in covariance structure analysis. American Statistics Association 1988. Proceedings of the Business and Economic Sections, (pp. 208-313).

Segars, A.H. \& Grover, V. (1993). Re-examining perceived ease of use and usefulness: a confirmatory factor analysis. MIS Quarterly 17 (4), (pp. 517-525).
Sharma, R.S., Siddiqui, A., Sharma, A., Sing, R., Kumar, R., Kaushal, S. et al.(2007). Leveraging knowledge management for growth: a case study of Tata consultancy services. Journal of Information Technology Case and Application Research, 9 (4), (pp. 29-65).

Smallborne, D., Leigh, R. \& North, D. (1995). The characteristics and strategies of high growth SMEs. International Journal of Entrepreneurial Behaviour \& Research, 1 (3), (pp. 44-62).

Smith, W.J. (1998). Turning toward growth. Empowerment in Organizations, 6 (4), (pp. 110-114).

Storey, D.J. (1994). Understanding the small business sector. London, UK: Routledge.

Sveiby, K.E. (1990). Knowledge management: 101 tips for leaders in knowledge-intensive organizations. Stockholm, Suecia: Affärsvärlden.

Sveiby, K.E. (1997). The new organizational wealth: managing and measuring knowledge based asset. San Francisco, USA: Barret-Koehler.

Ulrich, D. (1997). Human resource champion. Boston, USA: Harvard Business School Press.

Von Krogh, G., Nonaka, I. \& Aben, M. (2001). Making the most of your company's knowledge: a strategic framework. Long Range Planning, 34 (4), (pp. 421-439).

Watson, K., Hogarth-Scott, S. \& Wilson, N. (1998). Small business start-ups: success factors and support implications. International Journal of Entrepreneurial Behaviour \& Research, 4 (3), (pp. 217-238).

Weinzimmer, L.G. (2000). A replication and extension of organizational growth determinants. Journal of Business Research, 48 (1), (pp. 35-41).

Wilson, T.D. (2002). The nonsense of knowledge management. Information Research, 8 (1), (pp. 144-154).

Zack, M.H. (1999). Developing a knowledge strategy. California Management Review, 41 (3), (pp. 125-145).

Zhang, Y. (2000). Learning function and small business growth. Managerial Accounting Journal, 15 (5), (pp. 228-231). 